\title{
Real Space Cluster Expansion for Total Energies of Pd-Rich PdX (X = Rh, Ru) Alloys, Based on Full-Potential KKR Calculations: An Approach from a Dilute Limit
}

\author{
Chang Liu ${ }^{1}$, Mitsuhiro Asato ${ }^{2,3, *}$, Nobuhisa Fujima ${ }^{4}$, Toshiharu Hoshino ${ }^{5}$, Ying Chen ${ }^{6}$ and Tetsuo Mohri ${ }^{7}$ \\ ${ }^{1}$ National Institute for Materials Science, Tsukuba 305-0047, Japan \\ ${ }^{2}$ National Institute of Technology, Niihama College, Niihama 792-8580, Japan \\ ${ }^{3}$ Department of Chemistry and Biochemistry, University of California, Los Angeles, California 90095, United States \\ ${ }^{4}$ Graduate School of Engineering, Shizuoka University, Hamamatsu 432-8561, Japan \\ ${ }^{5}$ Faculty of Engineering, Shizuoka University, Hamamatsu 432-8561, Japan \\ ${ }^{6}$ Graduate School of Engineering, Tohoku University, Sendai 980-8579, Japan \\ ${ }^{7}$ Institute for Materials Research, Tohoku University, Sendai 980-8577, Japan
}

We study the accuracy and convergence of the real space cluster expansion (RSCE) for the internal energies in the free energies of Pd-rich $\operatorname{PdX}(X=R h, R u)$ alloys, being used to study the phase stability and phase equilibria of Pd-rich PdX alloys in fcc structure. In the present RSCE from a dilute limit, the $\mathrm{X}$ atoms of minor element are treated as impurities in Pd. The $n$-body interaction energies (IEs) among X impurities in Pd are determined uniquely and successively from the low body to high body, by the ab-initio calculations based on the full-potential KorringaKohn-Rostoker Green's function method (FPKKR) for the perfect and impurity systems (Pd-host and $\mathrm{X}_{n}$ in $\mathrm{Pd}, n=1 \sim 4$ ), combined with the generalized gradient approximation in the density functional theory. We show that the total energies of the ordered $\mathrm{Pd}_{3} \mathrm{X}(\mathrm{X}=\mathrm{Rh}, \mathrm{Ru}$; $\mathrm{X}$-concentration $=25 \%$ ) alloys in $\mathrm{L} 1_{2}$ structure, obtained by the screened FPKKR band calculations, are reproduced very well (within the error of $\sim 1 \mathrm{mRy}$ per atom) by the present RSCE including the 2-body IEs up to the 20th nearest neighboring pair $\left(\mathrm{X}_{2}\right)$ and the 3-body IEs up to the clusters $\left(\mathrm{X}_{3}\right)$ in the two cubic cells in fcc structure. We clarify the contribution from each term (of the $0 \sim 4$ body terms) in the RSCE to the total energies of Pd-rich PdX alloys, the distance dependence of the 2-body $\left(\mathrm{X}_{2}\right)$ IEs, and the cluster-size dependence of 3-body $\left(\mathrm{X}_{3}\right)$ and 4-body $\left(\mathrm{X}_{4}\right)$ IEs. It is also shown that the contribution from the $n$-body $(n=2 \sim 4)$ IEs becomes smaller and smaller with the increase in $n$ and that the contribution from the 4-body IEs is very small (less than $\sim 0.2 \mathrm{mRy}$ per atom). [doi:10.2320/matertrans.M2018194]

(Received June 13, 2018; Accepted August 6, 2018; Published September 14, 2018)

Keywords: KKR-Green's function method, GGA, Fermi-Dirac distribution, real space cluster expansion for total energy, phase stability, Pdrich PdRu alloys, Pd-rich PdRh alloys

\section{Introduction}

We recently succeeded in reproducing very well the observed X-concentration dependence of the solvus temperatures of the $\mathrm{Pd}$-rich $\mathrm{PdX}(\mathrm{X}=\mathrm{Ru}, \mathrm{Rh})$ alloys in fcc structure, ${ }^{1)}$ by the free energy calculations based on the cluster variation method (CVM) for the configurational entropy. ${ }^{2-5)}$ The internal energies in the free energies of the Pd-rich $\operatorname{PdX}(\mathrm{X}=\mathrm{Ru}, \mathrm{Rh})$ alloys, were calculated by the real space cluster expansion (RSCE) from a dilute limit, ${ }^{6-10)}$ where the $\mathrm{X}$ atoms of minor element were treated as impurities in Pd. The interaction energies (IEs) among $\mathrm{X}$ impurities in Pd were calculated by using the full-potential Korringa-Kohn-Rostoker Green's function method (FPKKR) for the perfect and impurity systems (Pd-host and $\mathrm{X}_{n}$ in $\mathrm{Pd}$, $n=0 \sim 4)$, combined with the generalized gradient approximation in the density functional theory. We have already clarified the distance dependence of the 2-body IEs, the local lattice relaxation effect, existing even at $T=0 \mathrm{~K}$, and both the temperature dependence of (1) the thermal lattice vibration effect by the Debye-Grüneisen model and (2) the electron excitation effect due to the Fermi-Dirac (FD) distribution. $^{3-5}$ ) We found that the temperature $\left(T_{\mathrm{FD}}\right)$ dependence of the electron excitation effect due to the FD distribution, being usually neglected, is very important to reproduce the observed solvus temperatures of the Pd-rich PdX alloys. The present RSCE may be very efficient to calculate the internal energies in the free energies of the

*Corresponding author, E-mail: asato@sci.niihama-nct.ac.jp alloys of decomposition type, such as the Pd-rich $\mathrm{PdX}$ $(\mathrm{X}=\mathrm{Ru}, \mathrm{Rh})$ alloys, although we need to examine the accuracy and convergence of the present RSCE.

Differently from the present RSCE approach, there are mainly two other methods to calculate the IEs for predicting the phase stability and phase equilibria in alloys: the approach based on the generalized perturbation method $(\mathrm{GPM})^{11)}$ using the Korringa-Kohn-Rostker coherentpotential approximation (KKR-CPA), ${ }^{12}$ and the ConnollyWilliams (CW) approach. ${ }^{13)}$ The GPM combined with the KKR-CPA method starts from the completely disordered states of alloys, ${ }^{12}$ ) described by the KKR-CPA, and then calculates the 2 4-body IEs by inserting 1 4 specific atoms in the CPA medium. The concentration dependence of the IEs is fully included as a result of the embedding into the CPA medium. However, the KKR-CPA relies on the single-site approximation being not unproblematic for alloys with charge transfer. ${ }^{14)}$ In addition to this approximation, the 2 4-body IEs are calculated in the frozen-potential approximation and moreover by taking only single-particle energies into account in the GPM. In the present RSCE approach, all these approximations are avoided, which has to be paid off by being restricted to the dilute limit. ${ }^{2-5}$ )

The CW approach determines the IEs by using the total energies of different atomic configurations in the considered alloys. Here the total energies obtained by the supercell calculations with many different configurations in the unit cell are fitted by selected models containing the $2 \sim 4$-body IEs. The best fit obtained by the smallest number of parameters determines the optional model for the IEs, which 
depends on both of the selections of models and supercells, and therefore cannot be determined uniquely, as discussed in Ref. 12. The present RSCE approach, being the most fundamental among these three approaches, considers only the atomic configurations of impurities, the energy differences of which define uniquely the $n$-body IEs among impurities. The $n$-body IEs are determined successively from the low body to high body and are independent on the impurity concentration, as discussed in Sect. 2. Because of an approach from a dilute limit, however, the efficiency of the present RSCE depends very much on the distance dependence of the 2-body ( $\mathrm{X}_{2}$ impurities) IEs and the clustersize dependence of the 3-body ( $\mathrm{X}_{3}$ impurities) and 4-body ( $\mathrm{X}_{4}$ impurities) IEs. However, we may usually expect that the interactions between distant sites are less important than those between near sites and that the interactions involving many sites are less important than the interactions involving the fewer sites. According to our experiences, the 2-body IEs are very small beyond the 10 -th $n n$ pair $^{3-5)}$ and the $n$-body IEs become smaller and smaller with the increase in $n$, and the 4-body IEs is very small compared with 2-body and 3-body IEs. ${ }^{2-5,7,8,10)}$

The purpose of the present paper is to clarify the accuracy and convergence of the present RSCE for the total energies of the $\operatorname{Pd}_{3} X(X=R u, R h)$ alloys in $\mathrm{L}_{2}$ structure, including up to the 4-body IEs. In Sect. 2, we describe the calculation procedures for the FPKKR with the electron excitation effect due to the FD distribution, and the RSCE from a dilute limit. In Sect. 3, we show how the FPKKR band calculation results for the total energies of the ordered $\mathrm{Pd}_{3} \mathrm{X}$ alloys (Xconcentration $=25 \%$ ) in $\mathrm{L}_{2}$ structure are reproduced very accurately (within the error of $\sim 1 \mathrm{mRy}$ per atom) by the present RSCE from a dilute limit $\left(\mathrm{X}_{n}\right.$ impurities in $\left.\mathrm{Pd}\right)$, including the 2-body IEs up to the 20th-nearest neighboring $(n n)$ pair $\left(\mathrm{X}_{2}\right)$ and the 3-body IEs up to the clusters $\left(\mathrm{X}_{3}\right)$ in the 2 cubic cells in fcc structure. We clarify each contribution from the $n$-body $(n=0 \sim 4)$ IEs in the RSCE, the distance dependence of the 2-body $\left(\mathrm{X}_{2}\right)$ IEs, and the cluster-size dependence of 3-body $\left(\mathrm{X}_{3}\right)$ and 4-body $\left(\mathrm{X}_{4}\right)$ IEs. It is shown that the contribution from the $n$-body $(n=2 \sim 4)$ IEs becomes smaller and smaller with the increase in $n$ and that the contribution from the 4-body IEs is less than $\sim 0.2 \mathrm{mRy}$ per atom. Section 4 summarizes the main results of the present paper and discusses the future problems.

\section{Calculation Method}

\subsection{Total energies of impurity systems}

The calculations for the total energy of $X(=R u, R u)$ impurities in $\mathrm{Pd}$ metal are based on the density functional theory (DFT) in the generalized gradient approximation (GGA). ${ }^{15,16)}$ In order to solve the Kohn-Sham equations we used a multiple scattering theory in the form of the KKR Green's function method for the full-potentials (FP). We used the screened version of the FPKKR band calculations for $\mathrm{Pd}$ host, which simplifies very much the numerical calculations by introducing the short-range structural Green's functions. ${ }^{17,18)}$ In order to simplify the total energy calculations in the GGA formalism, we used the electronic densities obtained self-consistently by the local spin density approximation in the DFT. The accuracy of the present GGA calculations was discussed in Refs. 15 and 16. The advantage of the Green's function method is that, by the introduction of the host Green's function, the embedding of point defects in an otherwise ideal crystal is described correctly, differently from the usual supercell and cluster calculations. It is noted that the change of the wave functions due to the defects is delocalized over the whole space, although the potential perturbation due to the defects is localized in the vicinity of the defects. The practical advantage in using the Green's function method is to exploit this short-range nature of the defect potential. For example, in order to obtain the accurate and converged total energies without the lattice distortion effect, for the impurity system in fcc structure, it may be enough to redetermine self-consistently only the potentials of impurities and their 1st- $n n$ host atoms, if the total energy change due to the perturbed wave functions over the infinite space is correctly evaluated by using the Lloyd's formula. ${ }^{7,16)}$ This is also very different from the supercell and cluster calculations where all potentials must be recalculated in each iteration cycle.

In the present method, we introduced the FD distribution into ab-initio Green's function calculations. As shown in Ref. 19, this is very useful to simplify the ground state calculations. Based on a contour integration in the complex energy plane we evaluate the residues at a few Matsubara frequencies, while the remaining integral is analyzed by the Sommerfeld expansion. The imaginary part $(\delta)$ in the complex energy plane is related to $T_{\mathrm{FD}}$ of the FD distribution $\left(\delta=\pi k T_{\mathrm{FD}}\right)$. The total energy is calculated by the grand canonical potential for which a simple formula is given. In all the present calculations, the lattice parameter of $\mathrm{Pd}$ host and the $T_{\mathrm{FD}}$ for the electron excitation due to the FDdistribution were fixed at 7.40 a.u. and $800 \mathrm{~K}$, respectively.

\subsection{Real space cluster expansion for total energies of Pd-rich PdX (X = Ru, Rh) alloys}

A characteristic feature of the present RSCE for total energies of Pd-rich PdX alloys is that all the terms in the present RSCE are uniquely determined by the combination of total energies in different atomic arrangements of $\mathrm{X}$ atoms in $\mathrm{Pd}$. It is noted that the $\mathrm{X}$ atoms of minor element are treated as impurities in Pd. In order to study the accuracy and convergence of the present RSCE, we calculate the total energies of the ordered $\operatorname{Pd}_{3} X(X=R u, R h)$ alloys in $\mathrm{L1}_{2}$ structure (Fig. 1, X-concentration $=25 \%$ ) and compare them with the band calculation results obtained by the screened FPKKR calculations for the ordered alloys. The RSCE for the total energy (per unit cell) of the ordered $\mathrm{Pd}_{3} \mathrm{X}$ alloy in $\mathrm{L}_{2}$ structure is written as follows:

$$
\begin{aligned}
E_{\mathrm{Pd}_{3} \mathrm{X}}^{\text {band }}= & 4 E_{\mathrm{Pd}}^{\text {band }}+\Delta E_{\mathrm{X} \text { in Pd }}^{1 \text {-body }}+\Delta E^{2 \text {-body }}+\Delta E^{3 \text {-body }} \\
& +\Delta E^{4-\text { body }}+\Delta E^{\text {many-body IEs beyond 4-body }}
\end{aligned}
$$

where the 1 st term is $4 \times$ total energy of Pd atom in Pd fcc metal (0-body), the 2 nd term the total energy change due to the insertion of a single X impurity in Pd fcc metal (1-body), the $3 \sim 5$ th terms correspond to the total contributions from the $n$-body $(n=2 \sim 4)$ IEs among $\mathrm{X}_{n}$ impurities in Pd fcc metal ( $2 \sim 4$ bodies), respectively, and the last term is the sum total of many-body IEs beyond the 4-body. The first 


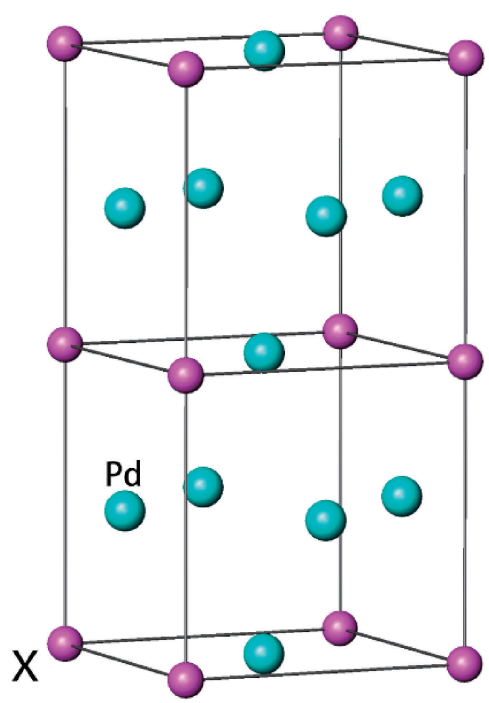

Fig. $1 \operatorname{Pd}_{3} \mathrm{X}(\mathrm{X}=\mathrm{Ru}, \mathrm{Rh})$ in $\mathrm{L1}_{2}$ structure.

(0-body) and second (1-body) energies are the quantities characteristic of $\mathrm{Pd}$-host and a single $\mathrm{X}$ impurity in $\mathrm{Pd}$, respectively. Thus, these components do not depend on the atomic arrangement of $\mathrm{X}$ impurities in Pd. It is noted that $\Delta E^{n \text {-body }}(n \geq 2)$ includes many kinds of $n$-body IEs. For example, $\Delta E^{2 \text {-body }}$ includes all the contributions from the 2-body interactions of the $(n, l, m)-n n$ pairs $(n, l, m=$ integer $)$ in unit of the lattice parameter of fcc structure, such as the 2nd $(1,0,0)-$, 4th $(1,1,0)-$, 6th $(1,1,1)-$, 8th $(2,0,0)$-, 10th $(1,2,0)-n n$ pairs, which exist in the ordered $\mathrm{Pd}_{3} \mathrm{X}$ alloy in $\mathrm{L}_{2}$ structure. $\Delta E^{3 \text {-body }}$ and $\Delta E^{4 \text {-body }}$ also include the contributions from the many 3-body and 4-body IEs of different shaped clusters (of $\mathrm{X}_{3}$ and $\mathrm{X}_{4}$ impurities) consisting of $(n, l, m)-n n$ pairs such as the 2nd-, 4th-, and 6th-nn pairs.

Now we explain the definition of the $n$-body IEs among $\mathrm{X}_{n}$ impurities in $\mathrm{Pd}$ and show how to calculate them. For example, we consider the $n$-body $(n=2 \sim 4)$ IEs within a square consisting of the four 2 nd- $n n$ pairs in $\mathrm{L1}_{2}$ structure, as shown in Fig. 2. This cluster is the smallest cluster for the 4-body IEs. Figures 2(a)-(f) show the six kinds of $\mathrm{Pd}_{4-n} \mathrm{X}_{n}$ square clusters including $\mathrm{X}_{n}(n=0 \sim 4)$ impurities in $\mathrm{Pd}$ fcc metal. There are two kinds of $\mathrm{X}_{2}$ pairs (the 2nd- and 4th- $n n$ pairs) designated by suffixes (2) and (4). Using the total energies $\left(E_{\mathrm{Pd}_{4}}, E_{\mathrm{X}_{1} \mathrm{Pd}_{3}}, E_{\mathrm{X}_{2} \mathrm{Pd}_{2}}{ }^{(2)}, E_{\mathrm{X}_{2} \mathrm{Pd}_{2}}{ }^{(4)}, E_{\mathrm{X}_{3} \mathrm{Pd}_{1}}\right.$ and $\left.E_{\mathrm{X}_{4}}\right)$ of these six kinds of clusters in Pd fcc metal, the binding energies of the $2 \sim 4$ impurities in Pd fcc metal are written as follows,

$$
\begin{aligned}
& B_{\mathrm{X}_{2} \mathrm{Pd}_{2}}{ }^{(2)}=E_{\mathrm{X}_{2} \mathrm{Pd}_{2}}{ }^{(2)}-2 E_{\mathrm{X}_{1} \mathrm{Pd}_{3}}+E_{\mathrm{Pd}_{4}} \\
& B_{\mathrm{X}_{2} \mathrm{Pd}_{2}}{ }^{(4)}=E_{\mathrm{X}_{2} \mathrm{Pd}_{2}}{ }^{(4)}-2 E_{\mathrm{X}_{1} \mathrm{Pd}_{3}}+E_{\mathrm{Pd}_{4}} \\
& B_{\mathrm{X}_{3} \mathrm{Pd}}=E_{\mathrm{X}_{3} \mathrm{Pd}_{1}}-3 E_{\mathrm{X}_{1} \mathrm{Pd}_{3}}+2 E_{\mathrm{Pd}_{4}} \\
& B_{\mathrm{X}_{4}}=E_{\mathrm{X}_{4}}-4 E_{\mathrm{X}_{1} \mathrm{Pd}_{3}}+3 E_{\mathrm{Pd}_{4}}
\end{aligned}
$$

It is noted that all the potentials in the 37-atom impurity region, including all the $\mathrm{Pd}$ host-atoms (totally $34 \mathrm{Pd}$ hostatoms) at the $1 \mathrm{st}-n n$ sites around a square cluster, are redetermined self-consistently in the present calculations. By using these binding energies, the 2 4-body IEs within the square cluster are defined as follows,

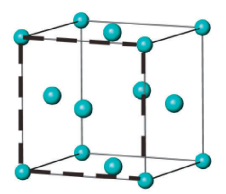

(a) 0-body

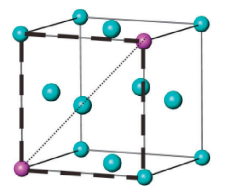

(d) 2-body (4)

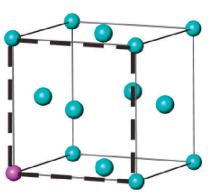

(b) 1-body

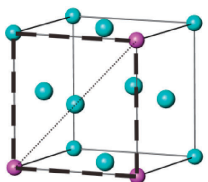

(e) 3-body

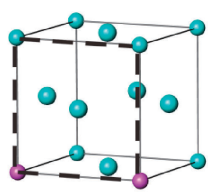

(c) 2-body (2) Pd(host)

$\mathrm{X}$ (impurity)

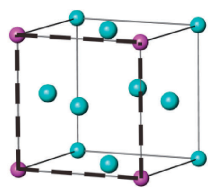

(f) 4-body
Fig. 2 Six kinds of square-shaped $\mathrm{Pd}_{4-n} \mathrm{X}_{n}(n=0 \sim 4)$ clusters in $\mathrm{Pd}$, shown by the broken lines. $(4-n) \times \mathrm{Pd}$ and $n \times \mathrm{X}$ atoms are located at vertices of a square consisting of the 2nd-nn pairs (a facet of fcc-cube). There are two kinds of pairs (2nd- and 4th-nn pairs).

$$
\begin{aligned}
\Delta E^{2 \text {-body }(2)}= & B_{\mathrm{X}_{2} \mathrm{Pd}_{2}}{ }^{(2)} \\
= & E_{\mathrm{X}_{2} \mathrm{Pd}_{2}}{ }^{(2)}-2 E_{\mathrm{X}_{1} \mathrm{Pd}_{3}}+E_{\mathrm{Pd}_{4}} \\
\Delta E^{2 \text {-body }(4)}= & B_{\mathrm{X}_{2} \mathrm{Pd}_{2}}{ }^{(4)} \\
= & E_{\mathrm{X}_{2} \mathrm{Pd}_{2}}{ }^{(4)}-2 E_{\mathrm{X}_{1} \mathrm{Pd}_{3}}+E_{\mathrm{Pd}_{4}} \\
\Delta E^{3 \text {-body }}= & B_{\mathrm{X}_{3} \mathrm{Pd}_{1}}-2 \Delta E^{2 \text {-body }(2)}-\Delta E^{2 \text {-body }(4)} \\
= & E_{\mathrm{X}_{3} \mathrm{Pd}_{1}}-2 E_{\mathrm{X}_{2} \mathrm{Pd}_{2}}{ }^{(2)}-E_{\mathrm{X}_{2} \mathrm{Pd}_{2}}{ }^{(4)} \\
& +3 E_{\mathrm{X}_{1} \mathrm{Pd}_{3}}-E_{\mathrm{Pd}_{4}} \\
\Delta E^{4-\text { body y }}= & B_{\mathrm{X}_{4}}-4 \Delta E^{3-\text { body }}-4 \Delta E^{2-\text { body }(2)}-2 \Delta E^{2 \text {-body }(4)} \\
= & E_{\mathrm{X}_{4}}-4 E_{\mathrm{X}_{3} \mathrm{Pd}_{1}}+4 E_{\mathrm{X}_{2} \mathrm{Pd}_{2}}{ }^{(2)}+2 E_{\mathrm{X}_{2} \mathrm{Pd}_{2}}{ }^{(4)} \\
& -4 E_{\mathrm{X}_{1} \mathrm{Pd}_{3}}+E_{\mathrm{Pd}_{4}}
\end{aligned}
$$

where the binding energy of 2 impurities corresponds to the 2-body IE. It is noted that the $n$-body IE is obtained by subtracting all the IEs up to $(n-1)$-body from the binding energy of $n$ impurities. The other $n$-body IEs of the cluster with the larger interatomic distances, needed for the RSCE of total energies of the ordered $\mathrm{Pd}_{3} \mathrm{X}$ in $\mathrm{L1}_{2}$ structure, can be calculated in the same way. Thus, the $n$-body IEs are determined uniquely and successively from the low body to high body and independent on the $\mathrm{X}$ concentration.

According to our experiences, the 2-body IEs are very small beyond the 10 -th $n n$ pair $^{3-5,10)}$ and the $n$-body IEs become smaller and smaller with the increase in $n$ and the total contribution from the 4-body IEs is very small compared with those from the 2-body and 3-body IEs. ${ }^{7,8,10)}$ Thus, in the present paper, we examine the 2-body up to the 20th- $n n$ and the 3-body IEs in two cubes in fcc and the 4-body IEs in one cube in fcc, as shown in Figs. 3 and 4, respectively. The numbers of the $n$-body $(n=2 \sim 4)$ IEs per atom are determined by considering the atomic arrangement in the ordered $\mathrm{Pd}_{3} \mathrm{X}$ alloy at $\mathrm{L1}_{2}$ structure, shown in Fig. 1 .

\section{Accuracy and Convergence of Real Space Cluster Expansion for Pd-Rich PdX (X = Ru, Rh) Alloys}

We discuss the accuracy and convergence of the present RSCE for the internal energies in the free energies of the Pdrich $\operatorname{PdX}(X=R u, R h)$ alloys. $\left.^{2-5}\right)$ We show how the total energies for the ordered $\mathrm{Pd}_{3} \mathrm{X}$ (X-concentration $=25 \%$, Fig. 1) alloys in $\mathrm{L}_{2}$ structure, obtained by the screened- 


\section{1 cube}

in fcc structure

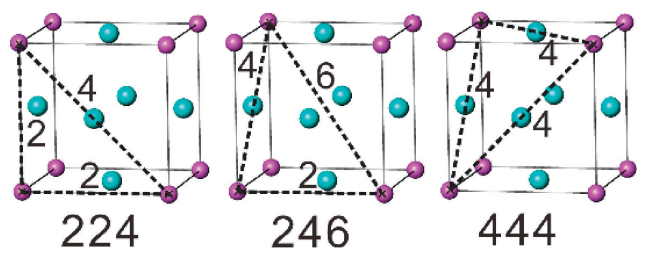

2 cubes

in fcc structure
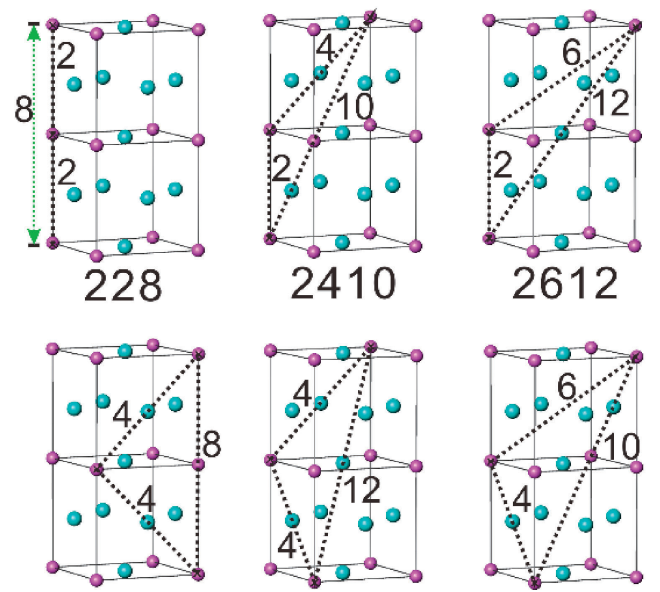

448

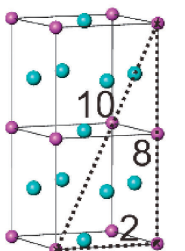

4412

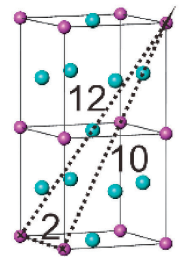

21012
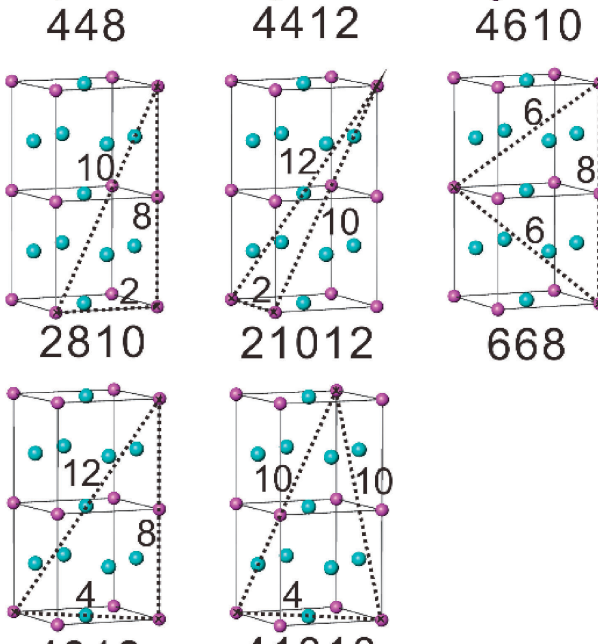

4812
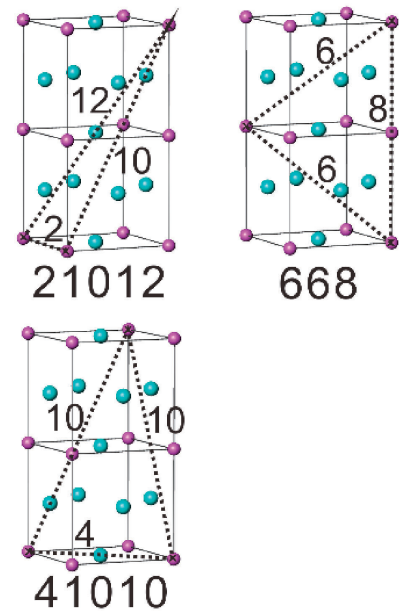

668

Fig. 3 Atomic arrangements for $\mathrm{X}_{3}$ clusters used in the calculations of the 3-body IEs in Pd-rich PdX alloys at $\mathrm{L}_{2}$ structure. There are three (eleven) kinds of clusters in one cubic cell (two cubic cells) in fcc structure. For example, the 224-cluster means that this cluster consists of two 2nd-nn and one 4th-nn pairs.

FPKKR band calculations, are reproduced by the present RSCE approach from a dilute limit (X impurities in $\mathrm{Pd}$ ). The calculated results for the ordered $\mathrm{Pd}_{3} \mathrm{X}$ alloys in $\mathrm{L}_{2}$ structure, including up to the 4-body IEs among $\mathrm{X}$ impurities in Pd, are listed in Table 1 (in unit of Ry), together with the FPKKR band calculation results (bottom line, described as "exact"). In the present RSCE from a dilute limit, we included the 2-body IEs up to the 20th- $n n$ pair and the 3-body and 4-body IEs of the clusters shown in Figs. 3 and 4. It is noted that the 1-body IE is the total energy change due to the insertion of a single X impurity, while the $n$-body $(n=2 \sim 4)$
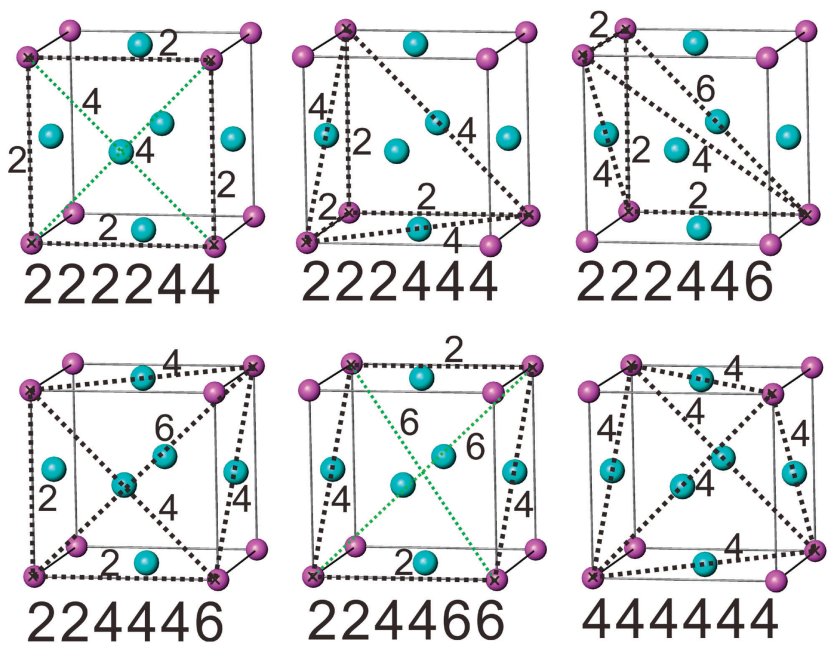

Fig. 4 Atomic arrangements for $\mathrm{X}_{4}$ clusters used in the calculations for the 4-body IEs in Pd-Rich PdX alloys at $\mathrm{L}_{2}$ structure. There are six kinds of clusters in one cubic cell in fcc structure. For example, the 222244-cluster means that this cluster consists of four 2 nd- $n n$ and two 4th- $n n$ pairs.

Table 1 Convergence of the RSCE for the total energies (in Ry) per unit cell (4 atoms) of (a) $\mathrm{Pd}_{3} \mathrm{Ru}$ and (b) $\mathrm{Pd}_{3} \mathrm{Rh}$ in $\mathrm{L1}_{2}$ structure. The values in parentheses indicate the contributions to the total energy in eq. (1), by the 1 st term $\left(4 \times E_{\mathrm{Pd}}^{\text {band }}\right)$, the 2 nd term $\left(\Delta E_{\mathrm{X} \text { in Pd }}^{1 \text {-body }}\right)$, and so on.

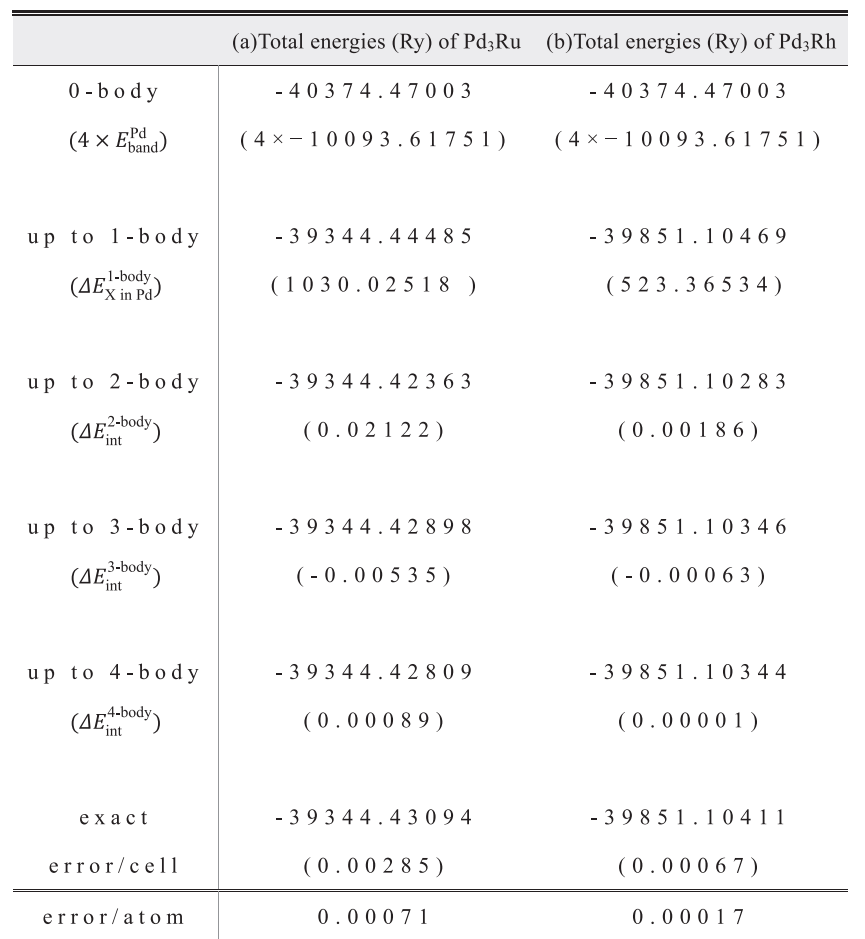

IEs are the total energy change due to the rearrangement of $\mathrm{X}_{n}$ impurities. Thus, the absolute value is very large for the 1-body IE, while very small for the $n$-body $(n=2 \sim 4)$ IEs, as listed in Table 1. We first show that the band calculation results can be reproduced very well (within the error of $4 \mathrm{mRy}$ per unit cell atom $(=1 \mathrm{mRy}$ per atom) $)$ by the RSCE including up to the 3-body IEs among $\mathrm{X}$ impurities in Pd.

For the $\mathrm{Pd}_{3} \mathrm{Rh}$ alloy, we found that the RSCE up to the 1-body term already reproduces very well (by the error of -0.59 mRy per unit cell) the band calculation result since the contribution from the 2 4-body IEs is very small, as listed 


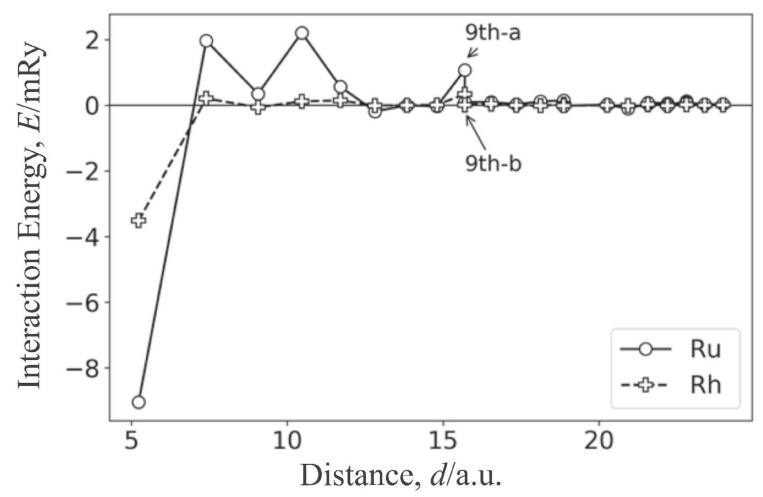

Fig. 5 Calculated results for the distance dependence (1 20th- $n n$ pairs) of $\mathrm{X}_{2}(\mathrm{X}=\mathrm{Ru}, \mathrm{Rh})$ IEs in Pd. There are two inequivalent sites for the 9th-, 13th-, 16th-, 17th-, and 18th-nn pairs. The 9th-a and 9th-b $n n$ sites are $(1.5,1.5,0)$ and $(2,0.5,0.5)$, in unit of the lattice parameter, respectively. The $(n, l, m) n n$ pairs (any two of $n, l, m$ are half-integer in unit of lattice parameter of fcc structure), such as 1 st $(0.5,0.5,0)-, 3$ rd $(1,0.5,0.5)-$, and 5th $(1.5,0.5,0)-n n$ pairs, do not exist in the ordered $\mathrm{Pd}_{3} \mathrm{X}$ alloy in $\mathrm{L1}_{2}$, as seen in Fig. 1 .

in Table 1 . The contribution from the $n$-body IEs in eq. (1) becomes as small as $1.86,-0.63$, and $0.01 \mathrm{mRy}$ per unit cell, with the increase in $n(=2 \sim 4)$. It is noted that the contribution from the 4-body IEs is almost zero. As results, the present RSCE including up to the 3-body IEs reproduce very accurately (by the error of $0.66 \mathrm{mRy}$ per unit cell) the band calculation result for the $\mathrm{Pd}_{3} \mathrm{Rh}$ alloy. The change of the RSCE error due to the inclusion of the contribution from the 4-body IEs is very small (from 0.66 to $0.67 \mathrm{mRy}$ per unit cell).

For the $\mathrm{Pd}_{3} \mathrm{Ru}$ alloy, the contribution from the 2 body IEs is as large as $21.22 \mathrm{mRy}$ per unit cell, as listed in Table 1 . However, the contribution from the $n$-body $(n=2 \sim 4)$ IEs in eq. (1) becomes as small as $21.22,-5.35$, and $0.89 \mathrm{mRy}$ per unit cell, with the increase in $n(=2 \sim 4)$. The total contribution from the 4-body IEs is less than $1 \mathrm{mRy}$ per unit cell $(=0.25 \mathrm{mRy}$ per atom $)$. As results, the present RSCE including up to the 3-body IEs reproduces very accurately (by the error of $1.96 \mathrm{mRy}$ per unit cell) the FPKKR band calculation results for the $\mathrm{Pd}_{3} \mathrm{Ru}$ alloys in $\mathrm{L}_{2}$ structure. The change of the RSCE error due to the inclusion of the contribution from the 4-body IEs is not large (from 1.96 to $2.85 \mathrm{mRy}$ per unit cell). It is noted that the RSCE error is less than $1 \mathrm{mRy}$ per atom for both the ordered $\mathrm{Pd}_{3} \mathrm{X}(\mathrm{X}=\mathrm{Ru}$, $\mathrm{Rh}$ ) alloys in $\mathrm{L1}_{2}$ structure, as listed in Table 1.

We now discuss the details of the contributions from the 2-body to the 4-body IEs. Figure 5 shows the distance dependence of the 2-body IEs (in unit of mRy), up to the 20th- $n n$ pairs for $\mathrm{X}_{2}(\mathrm{X}=\mathrm{Ru}, \mathrm{Rh})$ in $\mathrm{Pd}$. The calculated values for the 2-body IEs used in the RSCE are listed in Table 2. The positive value means repulsion, while the negative attraction. It is noted that the $(n, l, m)-n n$ pairs (any two of $n, l, m$ are half-integer) in unit of lattice parameter of fcc structure, such as 1 st $(0.5,0.5,0)-, 3 \mathrm{rd}(1,0.5,0.5)-$, and 5th $(1.5,0.5,0)-n n$ pairs, do not exist in the ordered $\mathrm{Pd}_{3} \mathrm{X}$ alloy in $\mathrm{L}_{2}$ structure, as seen in Fig. 1.

First, the attraction of the $1 \mathrm{st}(0.5,0.5,0)-n n$ pair is dominant, compared with the distant neighbor pairs, for both the impurity systems in Pd. The 2-body IEs beyond the 10th-
Table 2 Calculated results for the 2-body IEs (in mRy) of (a) $\mathrm{Ru}_{2}$ and (b) $\mathrm{Rh}_{2}$ impurities in $\mathrm{Pd}$, used in the RSCE of the total energies of ordered $\mathrm{Pd}_{3} \mathrm{X}(\mathrm{X}=\mathrm{Ru}, \mathrm{Rh})$ alloys in $\mathrm{L1}_{2}$ structure. The numbers of pairs per atom are also shown.

\begin{tabular}{c|c|cc}
\hline \hline 2-body & $\begin{array}{c}\text { Number of } \\
\text { pairs/atom }\end{array}$ & $\begin{array}{c}\text { (a) 2-body IEs (mRy) } \\
\mathrm{Ru}_{2} \text { in Pd }\end{array}$ & $\begin{array}{c}\text { (b) 2-body IEs (mRy) } \\
\mathrm{Rh}_{2} \text { in Pd }\end{array}$ \\
\hline $100(2 \mathrm{nd})$ & 3 & 1.9641 & 0.2580 \\
$110(4 \mathrm{th})$ & 6 & 2.2095 & 0.1312 \\
$111(6 \mathrm{th})$ & 4 & -0.1860 & 0.0038 \\
$200(8 \mathrm{th})$ & 3 & -0.0353 & 0.0095 \\
$210(10 \mathrm{th})$ & 12 & 0.0965 & 0.0398 \\
$211(12 \mathrm{th})$ & 12 & 0.1142 & -0.0092 \\
$220(15 \mathrm{th})$ & 6 & -0.1054 & -0.0095 \\
$221(17 \mathrm{th}-\mathrm{a})$ & 12 & 0.0617 & -0.0029 \\
$300(17 \mathrm{th}-\mathrm{b})$ & 3 & -0.0109 & -0.0101 \\
$310(19 \mathrm{th})$ & 12 & 0.0268 & 0.0009 \\
\hline
\end{tabular}

$n n$ pair are considerably small for both the impurity systems. The total contribution of the 2-body IEs up to the 10th- $n n$ is as large as $19.46(2.08) \mathrm{mRy}$ per unit cell for the total energy of $\mathrm{Pd}_{3} \mathrm{Ru}\left(\mathrm{Pd}_{3} \mathrm{Rh}\right)$ alloy, while that from the 11th- $n$ n to the 20th- $n n$ is as small as $1.76(-0.22)$ mRy per unit cell for the total energy of the $\mathrm{Pd}_{3} \mathrm{Ru}\left(\mathrm{Pd}_{3} \mathrm{Rh}\right)$ alloy. As results, the total contribution from the 2-body IEs is 21.22 (1.86) mRy per unit cell for $\mathrm{X}=\mathrm{Ru}(\mathrm{Rh})$, as listed in Table 1 .

Second, the characteristic feature for the distant neighbor interaction depends very much on $X$, as seen in Fig. 5. The 1 st- $n n$ interaction is much stronger for $\mathrm{X}=\mathrm{Ru}(-9.04 \mathrm{mRy})$ than for $\mathrm{X}=\mathrm{Rh}(-3.52 \mathrm{mRy})$. We have already shown in Ref. 2 that the $\mathrm{X}$-dependence $(\mathrm{X}=\mathrm{Zr}-\mathrm{Ag})$ of the 1 st-nn $\left(\mathrm{X}_{2}\right)$ IEs in $4 \mathrm{~d}$ fcc metals (Pd and $\mathrm{Ag}$ ) and $4 \mathrm{~d}$ bcc metals $(\mathrm{Nb}$ and Mo) is understood by the Friedel's band filling mechanism for d-states, in which the dependence of the band width on $\mathrm{X}$ metal is taken into account. In addition, the 2-body interaction is long-range for $\mathrm{X}=\mathrm{Ru}$ and considerably large repulsion for the 2 nd $(1,0,0)-$, 4th $(1,1,0)$-, and 9th-a $(1.5,1.5,0)-n n$ pairs, against the strong attraction of the 1 st$n n$ pair. $^{3)}$ On the other hand, the 2-body interaction is very short-range for $\mathrm{X}=\mathrm{Rh}$, although the interactions of the 2nd-, 4th- and 9th-a-nn pairs remains weakly repulsive. ${ }^{4}$ ) These results explain the fundamental feature of the experimentally known phase diagrams: ${ }^{1)}$ (1) the Pd-rich PdX (X = Ru, Rh) alloys are segregated at low temperature and disordered at high temperatures; (2) the solvus temperatures are much higher for $\mathrm{X}=\mathrm{Ru}$ than for $\mathrm{X}=\mathrm{Rh} .{ }^{3-5)}$ The disordered states become stable at high temperatures by the configurational entropy. It is noted that the atomic structures of alloys at low temperatures are determined by the minimization of the total sum of the small $n$-body IEs ( $n \geq 2)$, as discussed in Sec. 2.2, and that the total sum of the 2-body IEs may be most important for the atomic structures of alloys. We have already shown that the relative stability among the different atomic structures of the ordered Al-rich $\mathrm{AlX}(\mathrm{X}=\mathrm{Sc}-\mathrm{V})$ alloys and the stability of the atomic structures of AlMn quasicrystal and $\mathrm{Zr}_{70} \mathrm{Cu}_{30}$ bulk metallic glass are clarified by using the 2-body IEs. ${ }^{9,20,21)}$ It is also noted that the local lattice distortion effect 
Table 3 The same as Table 2 for the 3-body IEs (in mRy) of (a) $\mathrm{Ru}_{3}$ and (b) $\mathrm{Rh}_{3}$ impurities in $\mathrm{Pd}$. The numbers of clusters $\left(\mathrm{X}_{3}\right)$ per atom are also shown.

\begin{tabular}{|c|c|c|c|}
\hline 3-body & $\begin{array}{c}\text { Number of } \\
\text { clusters }\left(\mathrm{X}_{3}\right) / \text { atom }\end{array}$ & $\begin{array}{c}\text { (a) 3-body IEs (mRy) } \\
\mathrm{Ru}_{3} \text { in } \mathrm{Pd}\end{array}$ & $\begin{array}{c}\text { (b) 3-body IEs (mRy) } \\
\mathrm{Rh}_{3} \text { in } \mathrm{Pd}\end{array}$ \\
\hline 224 & 12 & -0.1609 & 0.0269 \\
\hline 246 & 24 & 0.0604 & 0.0231 \\
\hline 444 & 8 & 0.2540 & -0.0027 \\
\hline 228 & 3 & -0.2886 & -0.0138 \\
\hline 2410 & 24 & -0.0862 & -0.0034 \\
\hline 2612 & 24 & -0.0786 & -0.0062 \\
\hline 2810 & 24 & -0.0649 & -0.0135 \\
\hline 21012 & 48 & -0.0316 & -0.0052 \\
\hline 448 & 12 & 0.0395 & -0.0205 \\
\hline 4412 & 24 & 0.0966 & -0.0034 \\
\hline 4610 & 48 & -0.0264 & -0.0055 \\
\hline 4812 & 24 & -0.0075 & 0.0002 \\
\hline 41010 & 24 & 0.0076 & 0.0011 \\
\hline 668 & 12 & -0.0446 & -0.0065 \\
\hline
\end{tabular}

always exists in the disordered A-rich AX alloy and becomes usually high for $\mathrm{X}$ impurities with a large size-misfit (compared with A atom): ${ }^{3,4,22-24)}$ the distortion energy for the 2-body IEs in Pd is as large as $\sim-2.6 \mathrm{mRy}$ for $\mathrm{X}=\mathrm{Ru}$, and $\sim-0.9 \mathrm{mRy}$ for $\mathrm{X}=\mathrm{Rh}$ and becomes an important factor to reproduce the observed solvus temperatures of $\mathrm{Pd}$-rich $\mathrm{PdX}$ alloys, especially for $\mathrm{X}=\mathrm{Ru}$, as shown in Refs. 3 and 4

Table 3 shows the calculated results for the 3-body IEs (in unit of $m R y)$ of $X_{3}(X=R u, R h)$ impurity clusters in Pd. The atomic arrangements for the 3-body clusters $\left(\mathrm{X}_{3}\right)$ are shown in Fig. 3. For example, the 224-cluster means that this cluster consists of two 2 nd $(1,0,0)-n n$ and one 4th $(1,1,0)-n n$ pairs. The calculated results are summarized: (1) the 3-body IEs are smaller by one order of magnitude than the 2nd- $n n$ 2-body IEs (1.96 and $0.26 \mathrm{mRy}$ for $\mathrm{X}=\mathrm{Ru}, \mathrm{Rh})$, listed in Table 2, except for the three IEs $(-0.16,0.25,-0.29 \mathrm{mRy})$ of $224-$, 444-, 228-clusters for $X=R u$; (2) the 3-body IEs for $X=R h$ are much smaller than those for $\mathrm{X}=\mathrm{Ru}$, similarly to the 1 stnn 2-body IEs discussed above; (3) the 3-body IEs for the $\mathrm{X}_{3}$ clusters in the two cubic cells are usually very smaller than those for the $\mathrm{X}_{3}$ cluster in the one cubic cell, as being expected, although the 3-body IE of the 228-cluster in the two cubic cells is exceptionally large for $\mathrm{X}=\mathrm{Ru}$. It is noted however that the contribution of 228-cluster to the total energy of the $\mathrm{Pd}_{3} \mathrm{Ru}$ alloy in $\mathrm{L1}_{2}$ structure is not large because the number (3) of this cluster per atom is small, compared with those of the other $\mathrm{X}_{3}$ clusters, as listed in Table 3. It is also noted that the total contribution from the
Table 4 The same as Table 2 for the 4-body IEs (in mRy) of (a) $\mathrm{Ru}_{4}$ and (b) $\mathrm{Rh}_{4}$ impurities in $\mathrm{Pd}$. The numbers of clusters $\left(\mathrm{X}_{4}\right)$ per atom are also shown.

\begin{tabular}{c|c|cc}
\hline \hline 4-body & $\begin{array}{c}\text { Number of } \\
\text { clusters }\left(\mathrm{X}_{4}\right) / \text { atom }\end{array}$ & $\begin{array}{c}\text { (a) 4-body IEs (mRy) } \\
\mathrm{Ru}_{4} \text { in } \mathrm{Pd}\end{array}$ & $\begin{array}{c}\text { (b) 4-body IEs (mRy) } \\
\mathrm{Rh}_{4} \text { in } \mathrm{Pd}\end{array}$ \\
\hline 22244 & 3 & 0.1622 & 0.0289 \\
222444 & 8 & 0.0215 & 0.0028 \\
222446 & 24 & 0.0147 & 0.0005 \\
224446 & 24 & 0.0078 & -0.0076 \\
224466 & 6 & -0.0794 & 0.0067 \\
444444 & 2 & 0.0828 & 0.0179 \\
\hline
\end{tabular}

3-body IEs becomes considerably small by their cancellation due to the rapid oscillating character (between positive and negative values) of the 3-body IEs (see Table 3), depending on the cluster-size. As results, the total contribution from the 3-body IEs is as small as $-5.35(-0.63) \mathrm{mRy}$ per unit cell for $\mathrm{X}=\mathrm{Ru}(\mathrm{Rh})$, as listed in Table 1 . We have already shown that the sign and magnitude of the 3-body IEs are correlated with the shapes of the so-called Guinier-Preston (GP) zones of the age-hardening Al-rich $\mathrm{AlX}(\mathrm{X}=\mathrm{Cu}, \mathrm{Zn})$ alloys, such as the (001) disk in Al-rich $\mathrm{AlCu}$ alloys and the spherical shape in Al-rich AlZn alloys. ${ }^{6,8)}$

Table 4 shows the calculated results for the 4-body IEs (in unit of $\mathrm{mRy}$ ). The corresponding atomic arrangements for the 4-body clusters are shown in Fig. 4. For example, the 222244cluster means that this cluster includes the four 2 nd $(1,0,0)$ $n n$ pairs and two 4 th $(1,1,0)-n n$ pairs. The calculated values are considerably small except for the 4-body IEs $(0.16 \mathrm{mRy})$ of the 222244-cluster for $\mathrm{X}=\mathrm{Ru}$. The contribution of this 4-body IE to the total energy of the $\mathrm{Pd}_{3} \mathrm{Ru}$ alloy in $\mathrm{L1}_{2}$ structure is not large because the number (3) of this cluster per atom is small, as listed in Table 4. We may also expect the cancellation due to the oscillating character (between positive and negative values) of the small 4-body IEs (see Table 4), depending on the cluster-size. For example, for $\mathrm{X}=\mathrm{Ru}$, the contribution from the 222244 -cluster IEs $(0.1622 \times 3=$ $0.4866 \mathrm{mRy}$ ) is cancelled out by that from the 224466 -cluster IEs $(-0.0794 \times 6=-0.4776 \mathrm{mRy})$. As results, the total contribution from the 4-body IEs is as small as 0.89 (0.01) mRy per unit cell for $\mathrm{X}=\mathrm{Ru}(\mathrm{Rh})$, as listed in Table 1 .

\section{Summary and Future Problems}

We studied the accuracy and convergence of the present RSCE from a dilute limit, ${ }^{6-10)}$ which calculate the internal energies in the free energies of Pd-rich PdX alloys. In the present RSCE approach, the X atoms of minor element were treated as impurity atoms in $\mathrm{Pd}$. All the interaction energies (IEs) up to the 4-body among $\mathrm{X}$ impurities in Pd were determined uniquely and successively from the low body to high body by the FPKKR calculations for the perfect and impurity systems (Pd-host, $\mathrm{X}_{n}$ in $\mathrm{Pd}, n=1 \sim 4$ ). We found that (1) the interaction is considerably short-range for $\mathrm{Rh}$ impurities in $\mathrm{Pd}$, while long-range for $\mathrm{Ru}$ impurities in $\mathrm{Pd}$; (2) the 2-body interaction is strongly attractive for the 1st- $n n$ pair, compared with the weak repulsion at the 2nd-, 4th- and 
9th-a-nns; (3) the contribution from the $n$-body $(n=2 \sim 4)$ IEs becomes smaller and smaller with the increase in $n$ and the total contribution from the 4-body IEs is less than $1 \mathrm{mRy}$ per unit cell $(=0.25 \mathrm{mRy}$ per atom); (4) the FPKKR band calculation results for the ordered $\mathrm{Pd}_{3} \mathrm{X}(\mathrm{X}=\mathrm{Rh}, \mathrm{Ru}$; $\mathrm{X}$ concentration $=25 \%$ ) alloys in $\mathrm{L}_{2}$ structure are reproduced very well (within the error of $4 \mathrm{mRy}$ per unit cell $(=1 \mathrm{mRy}$ per atom)) by the present RSCE including the 2-body IEs up to the 20th $n n$ pair $\left(\mathrm{X}_{2}\right)$ and the 3-body IEs up to the clusters $\left(\mathrm{X}_{3}\right)$ in the two cubic cells in fcc structure. We also found that the total contribution from the 3-body IEs becomes considerably small by their cancellation due to the rapid oscillating character (between positive and negative values) of the 3-body IEs, depending on the cluster-size.

We have already shown in Refs. 3-5 that the present RSCE approach can take into account the lattice distortion effect (always existing in the disordered states) for the IEs among $\mathrm{X}$ impurities in $\mathrm{Pd}$, the temperature dependence of the FD distribution, and the thermal vibration effect by the Debye-Grüneisen model: the concentration dependence of the observed solvus temperatures of the Pd-rich $\mathrm{PdX}$ $(\mathrm{X}=\mathrm{Ru}, \mathrm{Rh})$ alloys were reproduced very well by the free energy calculations based on the present RSCE for the internal energies and the cluster variation method (CVM) for the configurational entropy. It is noted that the lattice distortion effect on the 2-body IEs of $\mathrm{X}(=\mathrm{Ru}, \mathrm{Rh})$ impurities in Pd works attractively and becomes important to reproduce the observed solvus temperatures of the Pd-rich PdX (X= $\mathrm{Ru}, \mathrm{Rh}$ ) alloys. ${ }^{3,4)}$

The present RSCE approach also allows us to study the stabilities of atomic structures in metallic alloys: the stabilities of atomic structures in Al-rich $\mathrm{AlX}(\mathrm{X}=\mathrm{Sc}-\mathrm{V}$, $\mathrm{Mn}$ ) alloys including AlMn quasicrystals ${ }^{9,20)}$ are clarified by the distance dependence of the 2-body IEs, while the shapes of GP zones in Al-rich AlX alloys by the sign and magnitude of 3-body IEs of the 1 st- $n n^{7,8)}$ Thus, the present RSCE approach may be very efficient in order to study the phase stability and phase equilibria of these metallic alloys.

We are now studying quantitatively the phase stability and phase equilibria of the age-hardenable Al-rich AlX (X $=\mathrm{Mg}$, $\mathrm{Cu}, \mathrm{Zn}$ ) alloys ${ }^{1)}$ by using the free energy calculations based on the present RSCE for the internal energy and the CVM for the configurational entropy. It is also very interesting to study the effect of another element addition on the kinetics of agehardening behaviors of Al-rich multi-element alloys, such as $\mathrm{Cu}$ in Al-rich AlMgSi alloys. ${ }^{25,26)}$ We plan to develop the calculation program for Monte Carlo (MC) simulations ${ }^{6,27-29)}$ which may be useful as one of material design tools for the high quality of age-hardenable Al-rich multi-element alloys. ${ }^{25)}$ For the mechanical properties of age-hardenable alloys, stability of precipitates is the central concern through nucleation, growth and coalescence processes. We believe Al-rich multi-element alloys offer a good example how thermodynamical calculations can reveal quantitative insight into the underlying age hardening behavior.

As another application of the present RSCE approach for the internal energy, it may be interesting to study the morphology in the nano-scale spinodal decomposition phase, ${ }^{30-32)}$ such as the magnetic network shown in Fig. 17 in Ref. 31, which may occur in the quenching and annealing process in the dilute magnetic semiconductors such as (Ga, Mn) N and (Ga, Mn)As. At present we can take into account the lattice distortion effect for the chemical and magnetic IEs derived from a dilute limit, ${ }^{6,33,34)}$ which may be important for Mn impurities (at Ga sites) in the GaAs (or $\mathrm{GaN}$ ) because the difference between the ionic radii (2.67 and 2.38 a.u.) of $\mathrm{Ga}$ and $\mathrm{Mn}$ atoms in fcc structure is considerably large. ${ }^{35)}$ However, in order to study the morphology, we must further develop, for example, the calculation program for MC simulations with the chemical and magnetic IEs. Morphological features expected by MC simulation with such intriguing energetics will be reported in the future.

\section{Acknowledgement}

The authors are grateful for the financial support from the Ministry of the Education, Culture, Sports, Science and Technology (JSPS KAKENHI Grant Nos. 15K06422 and 16K06710). This study was partly supported by New Energy and Industrial Technology Development Organization of Japan (NEDO) Grant (P16010). One of the authors (T. Mohri) appreciates their support. This study was almost completed when T. Hoshino was at the Institute for Material Research, Tohoku University. C. Liu and T. Hoshino would like to express their sincere thanks to the crew of the Center for Computational Materials Science of the Institute for Materials Research, Tohoku University, for their continuous support of the SR16000 supercomputing facilities.

\section{REFERENCES}

1) T.B. Massalski, H. Okamoto, P.R. Subramanian and L. Kacprazak: Binary Alloys Phase Diagrams, 2nd ed., (ASM International, New York, 1990).

2) M. Asato, T. Mizuno, T. Hoshino and H. Sawada: Mater. Trans. 42 (2001) 2216-2224

3) C. Liu, M. Asato, N. Fujima, T. Hoshino, Y. Chen and T. Mohri: Mater. Trans. 59 (2018) 338-347.

4) C. Liu, M. Asato, N. Fujima, T. Hoshino, Y. Chen and T. Mohri: Mater. Trans. 59 (2018) 883-889.

5) M. Asato, C. Liu, N. Fujima, T. Hoshino, Y. Chen and T. Mohri: World Academy of Science, Engineering and Technology International Journal of Materials and Metallurgical Engineering, Vol. 12, No. 3 (2018) pp. 119-123.

6) T. Hoshino, W. Schweika, R. Zeller and P.H. Dederichs: Phys. Rev. B 47 (1993) 5106-5117.

7) T. Hoshino, M. Asato, R. Zeller and P.H. Dederichs: Phys. Rev. B 70 (2004) 094118.

8) F. Nakamura, T. Hoshino, S. Tanaka, K. Hirose, S. Hirosawa and T. Sato: Trans. Mater. Res. Soc. Jpn. 30 (2005) 873-876.

9) M. Asato, H. Takahashi, T. Inagaki, N. Fujima, R. Tamura and T. Hoshino: Mater. Trans. 48 (2007) 1711-1716.

10) T. Hoshino, M. Asato and N. Fujima: J. Alloys Compd. 504 (2010) S534-S537.

11) F. Ducastelle and F. Gautier: J. Phys. F. 6 (1976) 2039.

12) For a review, see A. Gonis, M. Slutier, P.E.A. Turchi, G.M. Stocks and D.M. Nicholson: J. Less Common Met. 168 (1991) 127-144.

13) J.W. Connolly and A.R. Williams: Phys. Rev. B 27 (1983) 5169.

14) N. Stefanou, R. Zeller and P.H. Dederichs: Solid State Commun. 62 (1987) 735-738.

15) M. Asato, A. Settles, T. Hoshino, T. Asada, S. Blügel, R. Zeller and P.H. Dederichs: Phys. Rev. B 60 (1999) 5202-5210.

16) T. Hoshino, M. Asato, T. Mizuno and H. Fukushima: Mater. Trans. 42 (2001) 2206-2215

17) R. Zeller: Phys. Rev. B 55 (1997) 9400-9408

18) R. Zeller, M. Asato, T. Hoshino, J. Zalbloudil, P. Weinberger and P.H. 
Dederichs: Philos. Mag. B 78 (1998) 417-422.

19) K. Wildberger, P. Lang, R. Zeller and P.H. Dederichs: Phys. Rev. B 52 (1995) 11502-11508.

20) T. Hoshino, M. Asato, S. Tanaka, F. Nakamura and N. Fujima: Intermetallics 14 (2006) 913-916.

21) N. Fujima, M. Asato, R. Tamura and T. Hoshino: Mater. Trans. 48 (2007) 1734-1738

22) M. Asato, C. Liu, K. Kawakami, N. Fujima and T. Hoshino: Mater. Trans. 55 (2014) 1248-1256.

23) C. Liu, M. Asato, N. Fujima and T. Hoshino: Trans. Mater. Res. Soc. Jpn. 40 (2015) 159-164.

24) C. Liu, M. Asato, N. Fujima and T. Hoshino: Phys. Procedia 75 (2015) 1088-1095.

25) S. Hirosawa, F. Nakamura, T. Sato and T. Hoshino: J. Jpn. Inst. Light Met. 56 (2006) 621-628.

26) J. Kim, E. Kobayashi and T. Sato: Mater. Trans. 52 (2011) 906-913.

27) T. Hoshino, R. Zeller, P.H. Dederichs and T. Asada: Computational Physics as a New Frontier in Condensed Material Research, ed. by $\mathrm{H}$. Takayama et al., (Physical Society of Japan, Tokyo, 1995) pp. 105-
113.

28) W. Schweika: Disordered Alloys: Diffuse Scattering and Monte Carlo Simulations, 141, (Springer Tracts in Modern Physics, 1998) pp. 5157.

29) S. Hirosawa and T. Sato: J. Jpn. Inst. Light Met. 54 (2004) 121-127.

30) K. Sato, H. Katayama-Yoshida and P.H. Dederichs: Jpn. J. Appl. Phys. 44 (2005) L948-L951.

31) H. Katayama-Yoshida, K. Sato, T. Fukuyama, M. Toyoda, H. Kizaki, V.A. Dinh and P.H. Dederichs: J. Magn. Magn. Mater. 310 (2007) 2070-2077.

32) T. Dietl, K. Sato, T. Fukushima, A. Bonanni, M. Jamet, A. Barski, M. Tanaka, P.H. Hai and H. Katayama-Yoshida: Rev. Mod. Phys. 87 (2015) 1311-1377.

33) P. Lang, L. Nordstrom, K. Wildberger, R. Zeller, P.H. Dederichs and T. Hoshino: Phys. Rev. B 53 (1996) 9092-9107.

34) T. Hoshino, N. Fujima, M. Asato and H. Tatsuoka: J. Alloys Compd. 504 (2010) S531-S533.

35) C. Kittel: Introduction to Solid State Physics, 7th ed., (John \& Wiley Sons, Inc, New York, 1996) see Table 9. 